\title{
The clinical presentation of Legionella arthritis reveals the mode of infection and the bacterial species: case report and literature review
}

Marine Ibranosyan 1, Laetitia Beraud ${ }^{1,2}$, Hélène Lemaire ${ }^{3,4}$, Anne-Gaëlle Ranc ${ }^{1,2}$, Christophe Ginevra ${ }^{2,5,6,7}$, Sophie Jarraud $d^{1,2,5,6,7}$ and Ghislaine Descours ${ }^{1,2,5,6,7^{*}}$ (D)

\begin{abstract}
Background: While Legionella is a common cause of pneumonia, extrapulmonary infections like arthritis are scarce. Here, we describe a case of monoarthritis due to Legionella bozemanii, with no history of pneumonia. We provide a literature review of the 9 previously published Legionella arthritis and highlight a dichotomous epidemiology suggesting different physiopathological pathways leading to joint infection.

Case presentation: A 56-year old woman under immunosuppressive treatment by oral and intra-articular corticosteroids, methotrexate, and tocilizumab for an anti-synthetase syndrome was hospitalized for worsening pain and swelling of the left wrist for 3 days. Clinical examination showed left wrist synovitis and no fever. The arthritis occurred a few days after an accidental fall on wet asphalt responsible for a cutaneous wound followed by a corticosteroid intra-articular injection. Due to both the negativity of conventional culture of articular fluid and suspicion of infection, $16 \mathrm{~S}$ rRNA and specific PCRs were performed leading to the identification of L. bozemanii. Legionella-specific culture of the articular fluid was performed retrospectively and isolated L. bozemanii. The empiric antibiotic therapy was switched for oral levofloxacin and rifampin and the patient recovered after a 12-week treatment.

Conclusion: We report a case of L. bozemanii monoarthritis in an immunosuppressed woman, following a fall on wet asphalt and intra-articular corticosteroid injection. The review of the literature found that the clinical presentation reveals the mode of infection and the bacterial species. Monoarthritis more likely occurred after inoculation in patients under immunosuppressive therapy and were associated with non-Legionella pneumophila serogroup 1 (Lp1) strains that predominate in the environment. Polyarthritis were more likely secondary legionellosis localizations after blood spread of Lp1, the most frequently found in pneumonia. In both settings, $16 \mathrm{~S}$ rRNA and Legionella-specific PCR were key factors for the diagnosis.
\end{abstract}

Keywords: Legionella bozemanii, Arthritis, Immunosuppression, Corticosteroid, Tocilizumab, Methotrexate, Inoculation, 16S rRNA PCR, Extrapulmonary infections

\footnotetext{
* Correspondence: ghislaine.descours@univ-lyon1.fr

${ }^{1}$ Hospices Civils de Lyon, Groupement Hospitalier Nord, Institut des Agents

Infectieux, Laboratoire de Bactériologie, Lyon, France

${ }^{2}$ Hospices Civils de Lyon, Groupement Hospitalier Nord, Institut des Agents

Infectieux, Centre National de Référence des Légionelles, Lyon, France

Full list of author information is available at the end of the article
}

(c) The Author(s). 2019 Open Access This article is distributed under the terms of the Creative Commons Attribution 4.0 International License (http://creativecommons.org/licenses/by/4.0/), which permits unrestricted use, distribution, and reproduction in any medium, provided you give appropriate credit to the original author(s) and the source, provide a link to the Creative Commons license, and indicate if changes were made. The Creative Commons Public Domain Dedication waiver (http://creativecommons.org/publicdomain/zero/1.0/) applies to the data made available in this article, unless otherwise stated. 


\section{Background}

Legionnaires' disease (LD), a severe pneumonia caused by Legionella, represents $2-9 \%$ of cases of communityacquired pneumonia. There are more than 70 species and serogroups, but Legionella pneumophila serogroup 1 (Lp1) is responsible for more than $85 \%$ of cases of LD worldwide, while non-Lp1 infections occur more frequently in immunosuppressed patients [1]. Extrapulmonary infections, including arthritis, are scarce and not systematically related to pneumonia, suggesting a multifaceted infectious process. Herein, we report a case of $L$. bozemanii monoarthritis in an immunosuppressed woman, following a fall on wet asphalt and intra-articular corticosteroid (CS) injections.

\section{Case presentation}

A 56-year-old woman was hospitalized in the rheumatology department for worsening pain and swelling of the left wrist over the 3 preceding days. Her medical history included type 1 diabete and anti-synthetase syndrome diagnosed 1 year previously and which was characterized by polyarthritis, dermatomyositis and interstitial lung disease, and treated by prednisone $10 \mathrm{mg} /$ day, methotrexate (MTX) $20 \mathrm{mg} /$ week, and tocilizumab $560 \mathrm{mg} /$ month. During the 2 previous months, she suffered from stage-II synovitis of the left wrist predominant at radiocarpal and middle carpal joints, associated with flexor and extensor digitorum communis tenosynovitis; she received 2 intra-articular injections of triamcinolone 2 months and 3 days before admission.

On admission, the patient showed left wrist synovitis with no fever. Clinical examination found a wound of the 3rd proximal interphalangeal joint, related to an accidental fall on wet asphalt 4 days before. The blood count and C-reactive protein were normal. Chest X-ray showed no worsening of the interstitial lung disease. Blood samples were collected for culture. Over the following days, while she remained afebrile, local symptoms worsened, with onset of a painful epitrochlear lymphadenopathy. On day 4, a septic arthritis was suspected and aspiration of the left wrist fluid was performed; hemorrhagic fluid with a white blood cell count of 15.7 G/L and a neutrophil count of $8.0 \mathrm{G} / \mathrm{L}$ (51\%) was found. One sample was sent to the microbiology laboratory; Gram stain revealed no microorganism. An intravenous anti-staphylococcal antibiotic therapy (oxacillin $12 \mathrm{~g} /$ day, gentamicin $240 \mathrm{mg} /$ day) was initiated. On day 6, routine wrist fluid culture remained sterile, 16S rRNA [2] and Staphylococcus PCRs were negative, and blood cultures also remained sterile. On day 9, while the patient had improved, an inflammatory aspect of the back of the hand and severe pain persisted. The antibiotic spectrum was broadened to piperacillin-tazobactam $12 \mathrm{~g} /$ day and vancomycin $2 \mathrm{~g} /$ day on infectiologist advice. On day 14 , the patient had not improved and she underwent emergency surgery that found phlegmon of the F2 and F5 sheaths, requiring drainage and arthroscopic joint lavage. Six samples were sent to the microbiology laboratory; after 14 days of culture (day 28) a single Lysinibacillus fusiformis colony was found in 1 sample, and rare Cutibacterium acnes colonies in a second sample, which were considered as contaminants. On day 29, the $16 \mathrm{~S}$ rRNA PCRs were positive for Legionella spp. for 2 of the 4 sterile samples. The $23 \mathrm{~S}-5 \mathrm{~S}$ PCR performed on these samples identified $L$. bozemanii. The antibiotic regimen was modified to a 12-week course of oral levofloxacin 1 $\mathrm{g} /$ day and rifampin $1.2 \mathrm{~g} /$ day, and the symptoms quickly resolved. The immunosuppressive therapy by prednisone and MTX was continued Fig. 1.

Retrospectively, the joint fluid sampled on day 4 also tested positive for $L$. bozemanii by $23 S-5 S$ PCR. Five specimens (1 collected on day 4 , and 4 on day 14) were plated onto BCYE, BMPA and MWY plates (Oxoid, Dardilly, France). Only 1 day-14 sample grew L. bozemanii after 9 days. A $L$. bozemanii seroconversion $(<1 / 16$ before admission, $1 / 256$ on day 53) was demonstrated by in-house serology.

Regarding the source of infection, no clinical or radiological pneumonia was diagnosed before or during the course of the infection. L. bozemanii inoculation from asphalt at the time of her fall was strongly suspected. We hypothesized that the immunosuppressive treatment contributed to development of the infection and delayed diagnosis.

\section{Discussion and conclusions}

When examining this case alongside those previously published and summarized in Table $1(n=9)$ [3-11], it is of note that Legionella arthritis was mostly identified incidentally by $16 \mathrm{~S}$ rRNA PCR, which is consistent with the characteristics of the bacterium that does not usually grow on standard media. Interestingly, 2 clinical presentations can be distinguished. The first is monoarthritis $(n=7)$ that were all due to non-Lp1 strains and mainly reported in immunosuppressed patients $(n=6)$ with no history of pneumonia $(n=7)$; in 5 of the 7 cases, skin trauma close to the affected joint $(n=2)$ or an intraarticular injection or joint surgery $(n=3)$ was reported before arthritis. In contrast, cases of polyarthritis $(n=3)$ were all due to Lp1 and observed in patients with no immunosuppressive treatment who presented pneumonia at the time or before the diagnosis of arthritis. This dichotomous epidemiology suggests different physiopathological pathways leading to joint infection, which may result from both strain- and patient-related factors.

While more than 70 Legionella species have been isolated from freshwater and soil environments, less than half have been observed in clinical settings, and Lp1 is 


\section{1 year}

ago

Diagnosis of an anti-synthetase syndrome

(polyarthritis, dermatomyositis, interstitial lung

disease) treated by oral and intra-articular

corticosteroids, methotrexate, and tocilizumab

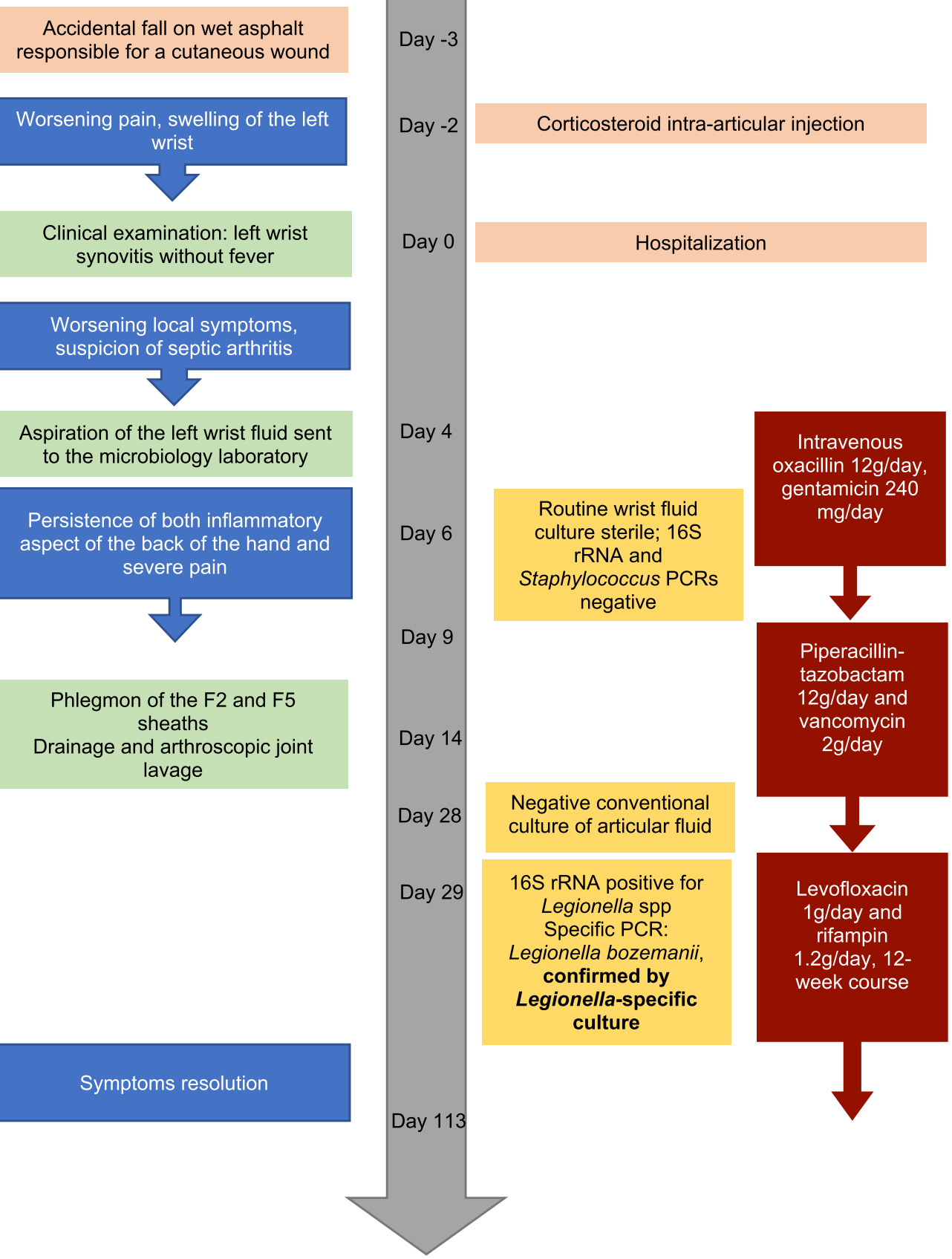

Resolution of the left wrist arthritis

Fig. 1 Timeline of interventions and outcomes 


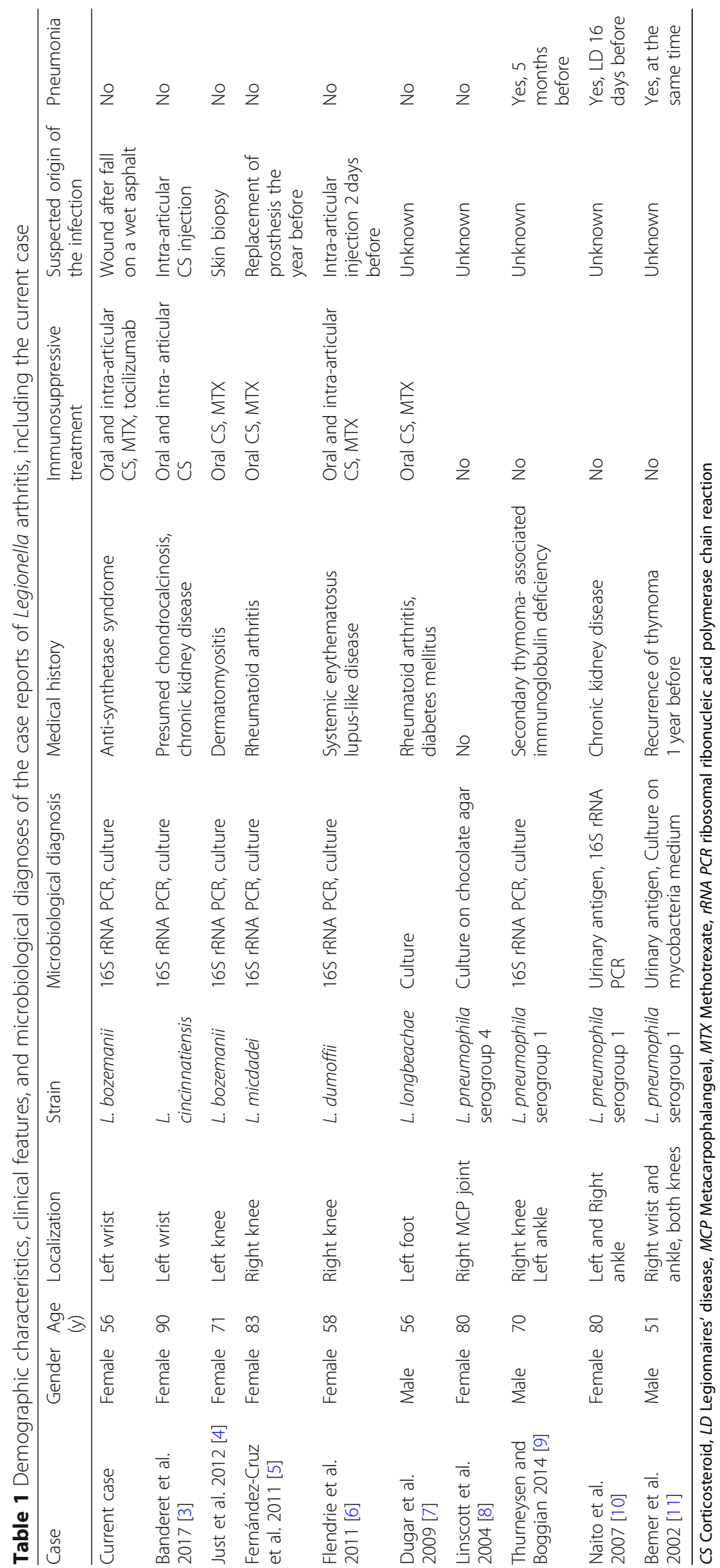


responsible for the vast majority of $\mathrm{LD}$ cases worldwide [1]. In contrast with pneumonia, non-Lp1 Legionella strains are predominant among the cases of monoarthritis reported in the literature (Table 1). This particular epidemiology is consistent with the environmental distribution of Legionella strains [12] and the direct mode of transmission from their natural niche; a similar epidemiology has been described for skin and soft tissue Legionella infections [13].

Host response to Legionella infections involves both innate and adaptive immunity $[14,15]$ and immunosuppressive therapies such as systemic CS, cytotoxic chemotherapies, and biological therapies (i.e. tumor necrosis factor inhibitors) are risk factors for Legionella infections [15-17]. Post-inoculation arthritis were described in patients receiving CS with or without MTX (Table 1), suggesting that inoculated Legionella was not cleared at the first step of infection. Three cases received intraarticular CS that constitutes both a gateway for environmental germs and an additional local risk factor for infection. In the case described herein, the patient also received tocilizumab, an IL-6 receptor antagonist. A case of Lp1 pneumonia in a patient under tocilizimab has been described [18]. As described herein, the patient presented no fever as tocilizumab inhibits IL-6, an inflammation and fever mediator [19], and, by delaying the diagnosis, tocilizumab contributes to uncontrolled infections.

Another clinical feature of Legionella arthritis is polyarthritis occurring during the course or after pneumonia. Interestingly, the reported cases are all due to Lp1 strains that predominate in LD (Table 1) suggesting secondary joint infections after bacterial blood dissemination. Accordingly, cases of Lp1 bacteremia have been described [20-22], and Lindsay et al. reported that up to $80.5 \%$ of patients present an Lp1 blood positive PCR after LD onset [23]. Nevertheless, non-Lp1 bacteremias have also been described [24, 25] and no data comparing blood bacterial loads between Lp1 and non-Lp1 strains are available.

In conclusion, the clinical presentation of Legionella arthritis reveals the mode of infection and orientates the microbiological diagnosis towards either Lp1 or nonLp1. In both contexts, $16 \mathrm{~S}$ rRNA and Legionella specific PCRs are key factors for the diagnosis.

\section{Abbreviations}

CS: Corticosteroid; LD: Legionnaires' disease; Lp1: Legionella pneumophila serogroup 1; MCP: Metacarpophalangeal; MTX: Methotrexate; rRNA

PCR: Ribosomal ribonucleic acid polymerase chain reaction

\section{Acknowledgements}

The authors are grateful to Dr. P Robinson for his critical reading of the manuscript.

\section{Authors' contributions}

MI drafted the manuscript. LB made substantial contributions to the analysis and interpretation of the data. HL, AGR, CG participated to acquisition of data. SJ designed the study and reviewed the manuscript for intellectual content. GD designed the study, made substantial contributions to the analysis and interpretation of the data and drafted the manuscript. All authors read and approved the final manuscript.

Funding

None.

Availability of data and materials

Not applicable.

Ethics approval and consent to participate

Not applicable.

\section{Consent for publication}

Written informed consent was obtained from the patient for publication of this case report. A copy of the written consent is available for review by the Editor of this journal.

\section{Competing interests}

The authors declare that they have no competing interests.

\section{Author details}

'Hospices Civils de Lyon, Groupement Hospitalier Nord, Institut des Agents Infectieux, Laboratoire de Bactériologie, Lyon, France. ${ }^{2}$ Hospices Civils de Lyon, Groupement Hospitalier Nord, Institut des Agents Infectieux, Centre National de Référence des Légionelles, Lyon, France. ${ }^{3}$ Hospices Civils de Lyon, Groupement Hospitalier Sud, Hôpital Lyon Sud, Service de Rhumatologie, Pierre-Bénite, France. ${ }^{4}$ Hospices Civils de Lyon, Groupement Hospitalier Centre, Hôpital Edouard Herriot, Service de Rhumatologie, Lyon, France. ${ }^{5} \mathrm{CIRI}$, Centre International de Recherche en Infectiologie, Equipe Pathogénèse des Légionelles, Lyon, France. ${ }^{6}$ Inserm, U1111, Université Lyon 1, CNRS, UMR5308, École Normale Supérieure de Lyon, Lyon, France. ${ }^{7}$ Univ Lyon 1, Lyon, France.

Received: 4 June 2019 Accepted: 20 September 2019

Published online: 21 October 2019

\section{References}

1. Cunha BA, Burillo A, Bouza E. Legionnaires' disease. Lancet. 2016;387:376-85.

2. Gazzano V, Berger A, Benito Y, Freydiere AM, Tristan A, Boisset S, et al. Reassessment of the role of rapid antigen detection tests in diagnosis of invasive group a streptococcal infections. J Clin Microbiol. 2016;54:994-9.

3. Banderet F, Blaich A, Soleman E, Gaia V, Osthoff M. Septic arthritis due to Legionella cincinnatiensis: case report and review of the literature. Infection. 2017:45:551-5.

4. Just SA, Knudsen JB, Uldum SA, Holt HM. Detection of Legionella bozemanae, a new cause of septic arthritis, by PCR followed by specific culture. J Clin Microbiol. 2012:50:4180-2.

5. Fernández-Cruz A, Marín M, Castelo L, Usubillaga R, Martín-Rabadán P, Bouza E. Legionella micdadei, a new cause of prosthetic joint infection. J Clin Microbiol. 2011;49:3409-10.

6. Flendrie M, Jeurissen M, Franssen M, Kwa D, Klaassen C, Vos F. Septic arthritis caused by Legionella dumoffii in a patient with systemic lupus erythematosus-like disease. J Clin Microbiol. 2011;49:746-9.

7. Dugar M, Rankin WA, Rowe E, Smith MD. "My foot hurts": a flare of rheumatoid arthritis? Med J Aust. 2009;190:392-3.

8. Linscott AJ, Poulter MD, Ward K, Bruckner DA. Legionella pneumophila Serogroup 4 isolated from joint tissue. J Clin Microbiol. 2004;42:1365-6.

9. Thurneysen C, Boggian K. Legionella pneumophila Serogroup 1 septic arthritis with probable endocarditis in an Immunodeficient patient. J Clin Rheumatol. 2014;20:297-8.

10. Naito T, Suda T, Saga K, Horii T, Chida K. Reactive Legionella pneumophila arthritis diagnosed by polymerase chain reaction. Rheumatol Int. 2007;27:415-6.

11. Bemer P, Leautez S, Ninin E, Jarraud S, Raffi F, Drugeon H. Legionella pneumophila arthritis: use of medium specific for mycobacteria for isolation of $L$. pneumophila in culture of articular fluid specimens. Clin Infect Dis. 2002;35:e6-7.

12. Doleans A, Aurell H, Reyrolle M, Lina G, Freney J, Vandenesch F, et al. Clinical and environmental distributions of Legionella strains in France are different. J Clin Microbiol. 2004;42:458-60. 
13. Padrnos LJ, Blair JE, Kusne S, Dicaudo DJ, Mikhael JR. Cutaneous legionellosis: case report and review of the medical literature. Transpl Infect Dis. 2014;16:307-14.

14. Newton HJ, Ang DKY, Van Driel IR, Hartland EL. Molecular pathogenesis of infections caused by Legionella pneumophila. Clin Microbiol Rev. 2010;23:274-98

15. Lanternier F, Ader F, Pilmis B, Catherinot E, Jarraud S, Lortholary O. Legionnaire's disease in compromised hosts. Infect Dis Clin N Am. 2017;31:123-35.

16. Carratala J, Gudiol F, Pallares R, Dorca J, Verdaguer R, Ariza J, et al. Risk factors for nosocomial Legionella pneumophila pneumonia. Am J Respir Crit Care Med. 1994;149:625-9.

17. Lanternier F, Tubach F, Ravaud P, Salmon D, Dellamonica P, Bretagne S, et al. Incidence and risk factors of Legionella pneumophila pneumonia during anti-tumor necrosis factor therapy: a prospective French study. Chest. 2013;144:990-8

18. Arinuma $Y$, Nogi S, Ishikawa Y, Nakayama H, Hashimoto A, Komiya A, et al. Fatal complication of Legionella pneumophila pneumonia in a Tocilizumabtreated rheumatoid arthritis patient. Intern Med. 2015;54:1125-30.

19. Netea MG, Kullberg BJ, Van der Meer JWM. Circulating Cytokines as Mediators of Fever. Clin Infect Dis. 2000;31(Supplement_5):S178-84.

20. Rihs JD, Yu VL, Zuravleff JJ, Goetz A, Muder RR. Isolation of Legionella pneumophila from blood with the BACTEC system: a prospective study yielding positive results. J Clin Microbiol. 1985;22:422-4.

21. Kaku N, Yanagihara K, Morinaga Y, Sato T, Nakashima M, Sakai T, et al. Detection of Legionella pneumophila serogroup 1 in blood cultures from a patient treated with tumor necrosis factor-alpha inhibitor. J Infect Chemother. 2013;19:166-70.

22. Lai CC, Tan CK, Chou CH, Hsu HL, Huang YT, Liao CH, et al. Hospitalacquired pneumonia and bacteremia caused by Legionella pneumophila in an immunocompromised patient. Infection. 2010;38:135-7.

23. Lindsay DSJ, Abraham WH, Findlay W, Christie P, Johnston F, Edwards GFS. Laboratory diagnosis of legionnaires' disease due to Legionella pneumophila serogroup 1: comparison of phenotypic and genotypic methods. J Med Microbiol. 2004:53:183-7.

24. Moriguchi S, Abe M, Kimura M, Yoshino C, Baba M, Okada C, et al. The diagnosis of Legionella pneumophila Serogroup 5 Bacteremic pneumonia during severe neutropenia using loop-mediated isothermal amplification. Intern Med. 2017;57:1045-8.

25. Cheung YF, Leung MP, Yuen KY. Legionella pneumonia and bacteraemia in association with protein-losing enteropathy after Fontan operation. J Inf Secur. 2001:42:206-7.

\section{Publisher's Note}

Springer Nature remains neutral with regard to jurisdictional claims in published maps and institutional affiliations.

Ready to submit your research? Choose BMC and benefit from:

- fast, convenient online submission

- thorough peer review by experienced researchers in your field

- rapid publication on acceptance

- support for research data, including large and complex data types

- gold Open Access which fosters wider collaboration and increased citations

- maximum visibility for your research: over $100 \mathrm{M}$ website views per year

At $\mathrm{BMC}$, research is always in progress.

Learn more biomedcentral.com/submissions 